\title{
Variations
}

Variations

Revue internationale de théorie critique

$17 \mid 2012$

Critique du travail

\section{Vivre une vie qui ne se vit pas. Quand Jean-Marie Vincent et André Gorz débattaient de valeur et subjectivité (2000-2002)}

Willy Gianinazzi

\section{OpenEdition}

Journals

Édition électronique

URL : http://journals.openedition.org/variations/354

DOI : $10.4000 /$ variations.354

ISSN : 1968-3960

Éditeur

Les amis de Variations

Référence électronique

Willy Gianinazzi, «Vivre une vie qui ne se vit pas. Quand Jean-Marie Vincent et André Gorz débattaient de valeur et subjectivité (2000-2002) », Variations [En ligne], 17 | 2012, mis en ligne le 15 octobre 2012, consulté le 20 avril 2019. URL : http://journals.openedition.org/variations/354 ; DOI : 10.4000/ variations.354

Ce document a été généré automatiquement le 20 avril 2019.

Les ami•e•s de Variations 


\title{
Vivre une vie qui ne se vit pas. Quand Jean-Marie Vincent et André Gorz débattaient de valeur et subjectivité (2000-2002)
}

\author{
Willy Gianinazzi
}

\section{Un compagnonnage de vieille date}

1 Peu après la disparition de Jean-Marie Vincent, en 2004, André Gorz a dit combien son ami avait été pour lui une personne importante ${ }^{1}$ : en 1959 elle l'avait initié à un pan de l'œuvre de Marx inconnu en France, puis mis en relation avec la gauche hétérodoxe italienne (sans doute les théoriciens Lelio Basso et Vittorio Foa). Alors que Gorz venait de publier son premier livre politique, La morale de l'histoire, à une époque où le marxisme occidental ne jurait que par les Manuscrits de 1844, Vincent le mit au courant de la parution à Berlin, six ans plus tôt, des Grundrisse. Ce texte, que Gorz citait dans cette édition d'origine, resta fondamental dans son œuvre. Il est un rien cocasse qu'il l'invoqua encore, dans le dialogue publié ici, pour contrer l'interprétation que son interlocuteur donnait de la valeur chez Marx.

Dans les années soixante, Vincent et Gorz étaient très liés. Ils partageaient une même sensibilité, celle du PSU, le premier en animant Tribune socialiste et en organisant des débats au Centre d'études socialistes, le second en devenant par ses écrits le théoricien du « réformisme révolutionnaire ». Mais leur évolution politique fut longtemps divergente : l'un fusionna son trotskisme autogestionnaire datant du PSU avec les vues stratégiques de la LCR avant de la quitter en 1981 et de faire fond sur les nouveaux sujets sociaux, alors que l'autre, cheville ouvrière des Temps modernes, fut proche de maoïstes en 68, puis devint le pionnier de l'écologie politique en France, qu'il féconda des idées d'Ivan Illich. Similairement, leur itinéraire philosophique se déroula sur des voies parallèles. Vincent 
devint le meilleur connaisseur en France du marxisme théorique italien (Galvano Della Volpe, Giulio Pietranera, Lucio Colletti) en faisant du fétichisme un outil de lecture du capital qui le préserva du "théoricisme » althussérien, en même temps qu'il devint un spécialiste de la sociologie allemande, en particulier de Max Weber et de l'école de Francfort. Gorz resta en bonne entente avec Vincent puisqu'au milieu des années soixante-dix, il publia, en tant que directeur de collection chez Galilée, un livre de Colletti postfacé par Vincent et l'ouvrage de celui-ci sur Horkheimer et Adorno. Mais son cheminement théorique fut différent. Imprégné de philosophie existentialiste, il fonda son marxisme sur la problématique de l'aliénation, en l'approfondissant cependant par une théorie des besoins, indépendante des thèses d'Adorno et anticipatrice de celle de l'école de Budapest, qui l'amena à se rapprocher d'une autre expression du francfortisme, celle « américaine » de Marcuse.

3 La véritable rencontre intellectuelle entre Vincent et Gorz eut lieu à la suite de la publication rapprochée de leurs ouvrages les plus ambitieux parmi ceux que chacun avait écrits jusque-là, respectivement Critique du travail (1987) et Métamorphoses du travail (1988). L'accord concernait le constat de la décentration du travail dans les procès subjectifs d'identification et d'intégration sociales. En réalité, sur ce point fondamental, leur convergence datait de la fin des années soixante-dix : avant que Gorz ne publie en 1980 ses iconoclastes Adieux au prolétariat, Vincent avait inauguré la nouvelle série de Critique de l'économie politique, qui ne faisait plus référence au programme de transition de la IV Internationale, par un article remarquable qui se terminait par ces mots : « Il n'y a plus de centralité de la production [...]. C'est seulement dans cette perspective qui n'est plus celle du travail que se mobiliseront tous ceux qui se révoltent aujourd'hui contre l'exploitation et l'oppression ou contre la domination d'un rapport social abstrait sur des individus dissociés et mutilés ${ }^{2}$.»

\section{Où est l'autonomie?}

4 Au cours des années quatre-vingt-dix, la revue Futur antérieur qu'animaient Vincent, son directeur, et quelques opéraïstes, dont Antonio Negri, se confronta à la pensée de Gorz et de manière d'autant plus pressante, voire polémique, que ce dernier se montrait de plus en plus réceptif aux thématiques de la revue et des théoriciens de l'« intellectualité de masse ». Le ralliement de Gorz, en 1996, au principe du revenu de citoyenneté comme moyen radical de développer l'autonomie existentielle en faisait un interlocuteur incontournable.

Le point de convergence/divergence portait précisément sur l'interprétation de la valeur dans le capitalisme contemporain où le capital fixe prend la forme prépondérante de l'immatériel en captant les facultés de connaissance et d'agir de la subjectivité. Il était entendu que le travail immédiat a perdu de son importance dans la mobilisation de tout ce qui concourt au procès de valorisation du capital et que donc le temps de travail ne peut plus être la mesure de la valeur. Mais est-ce que cela veut dire que la loi de la valeur disparaît parce que celle-ci est consubstantielle à son calcul par le temps ? Gorz répondit par l'affirmative, sur la base de l'utopisme marxien des Grundrisse, Vincent et Negri par un avis contraire, ayant pour eux une tradition affirmée de l'exégèse marxiste de la forme valeur fondée sur sa substance. La question pourrait paraître scolastique si on considère que tout le monde s'accordait pour dire que, valeur-travail ou pas, la domination du capital, ne pouvant plus compter sur l'étalon objectif du temps de travail sauf à titre 
d'invocation mystifiante au service du contrôle social, n'a jamais été aussi débridée, arbitraire et cynique. "La barbarie du calcul déréglé », comme disait Vincent ${ }^{3}$, fait que la résistance du capital à la diminution du temps de travail ne répond plus à une logique d'exploitation, mais à une volonté de domination qui la supplée. Une observation de Simone Weil, repérée jadis par Pierre Naville et Gorz, prend aujourd'hui tout son sens : « Nul n'accepterait d'être esclave deux heures; l'esclavage, pour être accepté, doit durer assez chaque jour pour briser quelque chose dans l'homme ${ }^{4}$. "Vincent le pensait aussi : «Les "managers" subodorent qu'une réduction systématique de la durée de travail peut à terme faire apparaître des aspirations fortes à une temporalité choisie chez les salariés ${ }^{5}$."

Et la subjectivité dans tout cela? Car c'est bien elle qui faisait débat et condensait les vrais clivages. Dans le «Dialogue » avec Gorz, que Vincent avait sollicité par questionnaire pour ouvrir le premier numéro de Variations, il était principalement question de l'évaluation de la valeur, mais la correspondance entre eux qui suivit cet échange prolongea tout naturellement la problématique en examinant de près la «subjectivité » dans son rapport ambivalent de soustraction et de soumission au procès de reproduction du capital. Au bout du compte, les lettres des deux amis montrent qu'ils n'étaient pas loin de s'accorder sur une définition de la subjectivité (ou sujet) comme expérience vécue et ancrée dans les dilemmes affectifs de l'individualité prise en tenailles entre soi et les autres, entre sa volonté de vivre et ce qui l'empêche de vivre. C'est pourquoi, de son côté, Gorz regardait avec sympathie les tentatives des disciples de Guattari (à distinguer ici de Deleuze) pour promouvoir une politique écologiste transversale, appelée "écosophie ", réceptive aux affects et aux façons de vivre ${ }^{6}$. De sorte que l'un et l'autre reprochaient finalement à Deleuze, Negri et à ses partisans, tout comme à Habermas, une vision mutilée de la subjectivité - pas assez inscrite dans la sensibilité et l'affect pour Vincent, pas assez imprégnée d'expérience sensible et de sens pour Gorz: en un mot, pas assez radicale dans sa négativité.

7 La question de savoir où se situe la subjectivité est un préalable pour évaluer sa puissance de négation. Pour Gorz considérer la subjectivité, à la manière de Negri, comme uniquement et forcément un agencement de l'intellectualité de masse à l'intérieur de la production immatérielle revenait à nier la possibilité d'en faire un levier de sécession tant son asservissement au commandement du capital, lui semblait-il, est immense ${ }^{7}$. Cette façon de voir était d'autant plus problématique à ses yeux que les rapports capitalistes s'étendent de plus en plus au-delà de l'économie productive en investissant de nouveaux domaines de la société (comme les services à la personne). Que devient dans un tel contexte l'aspiration des individus à l'autonomie?

8 Les enjeux politiques recelés par cette interrogation étaient d'actualité non seulement depuis qu'avait été publié, en 1995, pour le compte du Commissariat au Plan, le rapport Boissonnat sur les contrats de multiactivité, qui auraient asservi un peu plus la société à la logique économique (par les problèmes soulevés, un parallèle peut être fait aujourd'hui avec le statut de l'autoentrepreneur en France et en Italie), mais aussi parce qu'en ces années, nombre de penseurs et de militants se mobilisaient autour d'un Appel européen pour une citoyenneté et une économie plurielles (signé par Gorz, Negri, les animateurs du Mauss, de Transversales et tutti quanti) et enfin parce que les mouvements de chômeurs ( Partage, AC) n'étaient pas en reste pour proposer leur solution.

Compter sur la promotion des activités de tiers secteur (hors secteur marchand et service public) impliquait donc de réfléchir à ce qu'est la subjectivité dans son rapport essentiel à l'autonomie. On a compris que pour Negri, et certains de ses amis, la subjectivité 
n'advient et ne crée son sens que par la praxis productive mettant en mouvement l'intelligence générale et la puissance affective des individus coopérant entre eux ${ }^{8}$. C'est donc de l'intérieur du rapport au capital que l'autonomie peut acquérir ses marges. Mais, pour Vincent, il est possible « de faire sortir les subjectivités des prisons dans lesquelles les enferme la valorisation » seulement parce que l'homme qui entre dans la production n'est pas coupé « de ce qu'il fait comme être sexué dans des relations interindividuelles, comme participant à des réseaux d'interaction, comme organisateur de relations vitales » : la production n'est en définitive qu'un moment parmi d'autres dans la vaste dynamique des rapports sociaux'. Pour Gorz, plus drastiquement, la subjectivité capable d'autonomie et de mise en cause du sens et du but du travail commandé ne peut se constituer qu'en se soustrayant à l'emprise du capital au niveau de la société où ont lieu les expérimentations d'autoproduction et d'échanges gratuits, et où est menée la bataille culturelle, morale et politique ${ }^{10}$. De plus, ontologiquement parlant, le sujet ne «se fait " que dans la non-coïncidence et la résistance à sa socialisation fonctionnelle, de sorte qu'une activité, pour qu'elle soit réellement autonome, ne doit avoir pour fin qu'elle-même (ce n'est pas Kant mais Aristote et Marx que Gorz invoquait) ${ }^{11}$. Il était donc question d'organiser « l'exode » de la société de travail.

Tout cela, remarquait Vincent, "n'est évidemment possible que si l'on déconnecte dépense de la force de travail et rémunération ${ }^{12} »$. Aussi l'allocation universelle, qui casse par son inconditionnalité le rapport entre revenu et emploi, ajoutée à la réduction du temps de travail représentent-elles, aux yeux de Gorz ${ }^{13}$, un double outil pour développer des activités réellement autonomes à même de contrecarrer, moins en séparation qu'en hybridation dans la vie de l'individu, le travail encore rivé à la reproduction du capital. Vaste programme, qui demeure sur le métier...

\section{NOTES}

1. A. Gorz, Écologica, Paris, Galilée, 2008, p. 9-10 (entretien réalisé en 2004).

2. J.-M. Vincent, «La domination du travail abstrait », Critique de l'économie politique, 1, oct.-déc. 1977, p. 49.

3. J.-M. Vincent, « Sortir du travail », Futur antérieur, 43, [avr.] 1998, p. 87.

4. S. Weil, La condition ouvrière, Paris, Gallimard, 1951, p. 253.

5. J.-M. Vincent, « Sortir du travail », art. cit., p. 89.

6. Dans les lettres, Gorz citait Valérie Marange. Signalons un récent numéro de Chimères $(76,2011)$ consacré à l'écosophie qui comprend le bel article de Maud Granger Remy, « André Gorz et la poésie du monde vécu ».

7. Negri, de son côté : «Comment [Gorz] peut-il ne pas comprendre que c'est à partir de cette profondeur de l'insertion de la force de travail dans le capital que tout futur prendra forme? » (T.N., J.-M. V., « Paradoxes autour du travail », Futur antérieur, 10, 1992, p. 7 ; en cosignant ce texte, manifestement rédigé par Negri, le directeur de la revue lui apportait une caution, mais valait-elle adhésion? Nous ne saurions trancher). 
8. Citons, parmi les articles parus dans Futur antérieur, celui limpide de Pascal NicolasLe Strat, «Travail et constitution du sens. À propos d'André Gorz » $(35-36,1996)$. Pour Toni Negri, voir en particulier: "Travail et affect» $(39-40,1997)$. Negri a réitéré sa critique de Gorz, qu'il avait formulée dans son compte rendu de Misères du présent, richesse $d u$ possible $(43,1998)$, dans un tout récent entretien avec Carlo Vercellone et Christophe Fourel publié dans C. Fourel (ed.), André Gorz. Un penseur pour le XXI ${ }^{e}$ siècle, Paris, La Découverte, $2^{\mathrm{e}}$ éd. augm., 2012, p. 189-197.

9. J.-M. Vincent, « La déstabilisation du travail », Futur antérieur, 35-36, [sept. ] 1996, p. 29.

10. Voir, entre autres, son entretien avec Carlo Vercellone, Patrick Dieuaide et Pierre Peronnet dans Alice, 1, sept. 1998.

11. Voir A. Gorz, Métamorphoses du travail. Quête du sens. Critique de la raison économique, Paris, Galilée, 1988, p. 205-211.

12. J.-M. Vincent, « Sortir du travail », art. cit., p. 95.

13. A. Gorz, Misères du présent, richesse du possible, Paris, Galilée, 1997.

\section{INDEX}

Mots-clés : critique du travail, correspondance, artistes, socialisme vert, travailleurs culturels, Wertkritik, Gorz André, dialogue, Vincent Jean-Marie, écologie, modèles alternatifs, Marx Karl, penser le Capital, ethos du travail, Eglise Orthodoxe, salariat, domination

\section{AUTEUR}

\section{WILLY GIANINAZZI}

Historien, spécialiste par ailleurs de Georges Sorel et du syndicalisme révolutionnaire 